Check for updates

Cite this: RSC Adv., 2019, 9, 844

Received 10th September 2018 Accepted 19th December 2018

DOI: $10.1039 / c 8 r a 07533 f$

rsc.li/rsc-advances

\section{Applications of self-assembling ultrashort peptides in bionanotechnology}

\begin{abstract}
Ming $\mathrm{Ni}^{* a b}$ and Shuangmu Zhuo (D) *a
Peptides are intriguing building blocks for a variety of applications in bionanotechnology. Peptides can selfassemble into well-ordered nanostructures. Among the various nanomorphology forms, peptide nanofibers and nanotubes are relevant in biomedical applications. In this review, their applications as tissue engineering scaffolds, drug delivery vehicles, three-dimensional printing bioinks and bioimaging nanoprobes will be illustrated. This review article describes di-, tri-, tetra-peptides because they are cost-effective, simple to prepare, and amenable to production on a large scale.
\end{abstract}

\section{Introduction}

Peptides, both natural and synthetic, are amino acids linked by peptide bonds. They play important roles in many

${ }^{a}$ Fujian Provincial Key Laboratory for Photonics Technology, Key Laboratory of Optoelectronic Science and Technology for Medicine of Ministry of Education, Fujian Normal University, Fuzhou, 350007, P. R. China. E-mail: shuangmuzhuo@gmail. com; mingni.sg@gmail.com

${ }^{b}$ School of Biological Sciences \& Engineering, Yachay Tech University, Hacienda San José s/n, San Miguel de Urcuquí 100105, Ecuador biological processes, acting as biological catalysts, biological messengers, chemical messengers, and intracellular and intercellular mediators. Some peptides such as vasopressin, which are enzymes, are biological catalysts. Other peptides such as insulin, which are hormones, are biological messengers. Their unique biological functions may be defined by their specific structure. Peptides are intriguing building blocks to form nanostructures with distinct biological functions. For example, type I collagen is an important component of extracellular matrix (ECM). Its building blocks are G-P-X or G-XO. ${ }^{1}$ These peptide building blocks form triple helix nanofiber

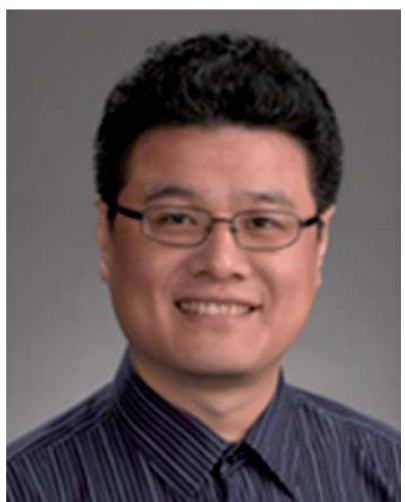

Dr Ming Ni received his doctoral degree in Chemical Engineering from the University of Washington in 2004. Under the supervision of Dr Buddy Ratner, his PhD project was to study the mechanism of osteoinduction by nacre (nacre also known as mother of pearl). After his graduation, he moved to Philadelphia and did two-year postdoc in Bioengineering at the University of Pennsylvania. Under the supervision of Dr Paul Ducheyne, his project was to design, synthesize and characterize a novel sol-gel derived, nanostructured material that used for drug delivery applications. In 2007, he moved to Singapore, working as a research scientist at the Institute of Bioengineering and Nanotechnology (IBN), Agency for Science, Technology and Research (A*STAR). Currently, he is an assistant professor at Yachay Tech University, located at Urcuqui, Ecuador. His current research focuses on biomaterials. He is also interested in advanced scanning optical microscopy, microfluidics, stem cell therapy and drug delivery.



Shuangmu Zhuo received the PhD degree in Optics Engineering from the Fujian Normal University, China, in 2012. He then joined the Singapore-MIT Alliance for Research and Technology as a Postdoctoral Research Fellow. He is currently a Professor in the College of Photonic and Electronic Engineering, Fujian Normal University, China. His research interests include the development and applications of nonlinear optical microscopy in biological and biomedical research. 
structures, which enable collagen to achieve high mechanic strength. Besides nanofibers, peptides can self-assemble into many forms of well-ordered nanostructures via non-covalent interactions. These interactions including electrostatic interactions, hydrogen bonding interactions, hydrophobic interactions, van der Waals interactions, and $\pi-\pi$ stacking interactions. The well-ordered nanostructures can be nanobelts, ${ }^{2}$ nanodoughnuts, ${ }^{3}$ nanofilaments, ${ }^{4}$ nanoparticles, ${ }^{5}$ nanorods, ${ }^{6}$ nanoropes, ${ }^{4}$ nanotubes, ${ }^{7}$ and nanovesicles ${ }^{8}$ (Fig. 1). Depending on their amino acid sequence and nanostructure, peptides may perform different biological functions. Peptide self-assembly can form hydrogels either by physical entanglement or chemical crosslinking. These self-assembled, nanostructured hydrogels are of great interest as biomaterials for applications in cell culture, drug delivery, immuneengineering, regenerative medicine and tissue engineering. ${ }^{9}$ Furthermore, peptide based nanomaterials are used as probes for bioimaging applications. ${ }^{\mathbf{1 0 - 1 4}}$ Based on these, researchers are interested in studying peptide-based biomaterials, especially for their applications in bionanotechnology.

Among all the peptide based biomaterials, ultrashort $(\leq 4$ amino acids) peptides are of great interest in recent years because they are cost-effective, simple to prepare, and amenable for production on a large scale. In this review, we describe several types of linear ultrashort peptides (dipeptides, tripeptides and tetrapetides). These ultrashort peptides can self-assemble into well-ordered nanostructures, such as nanotube or nanofiber, then form a three-dimensional network, such as hydrogels. These ultrashort peptide hydrogels can then be applied in bionanotechnology as bioimaging probes, bioprinting inks, cell culture scaffolds and drug delivery vehicles (Fig. 2).

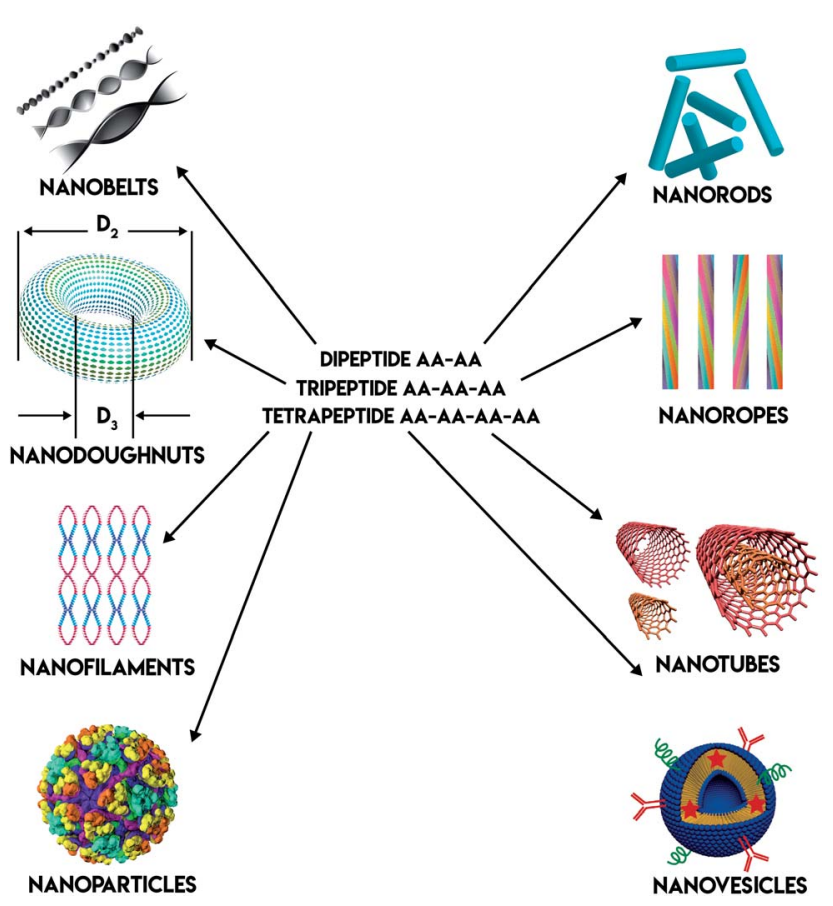

Fig. 1 Different peptide nanostructures.

\section{Dipeptides}

\section{Diphenylalanine (FF) nanostructures}

FF has been suggested to be the core recognition motif of the $\beta$ amyloid polypeptide. It has been well studied as an ultrashort peptide for decades. Reches and Gazit first reported the synthesis of FF nanotubes. ${ }^{7}$ The dipeptide building blocks selfassemble into nanotubes possibly by $\pi-\pi$ stacking and $\beta$-sheet secondary structure formation. First, researchers use it as a model to study amyloid aggregation. Later on, other applications of FF nanotubes in bionanotechnology were exploited. These nanotubes are chemically and thermally stable, having extraordinary mechanical strength. They have been used as a mold to cast silver nanowires, which enables its applications in the bioelectrical field. ${ }^{7}$ Gazit and coworkers ${ }^{15}$ achieved twodimensional nanotube arrays (nanoforest) with either vertical or horizontal patterns. Vertical patterns of FF nanotube arrays were achieved via rapid evaporation. Horizontal patterns of FF nanotube arrays were achieved by first coating FF nanotubes with magnetic particles and then applying an external magnetic field. FF nanotubes have unique optical properties. Kol et al. ${ }^{13}$ found FF nanotubes illuminate blue fluorescence in 2005 . Handelman et al. ${ }^{12}$ found $\mathrm{FF}$ nanotubes show second-order nonlinear optical response in 2013. These unique optical properties enable FF nanotubes to be used for nanophotonic devices. Moreover, cationic FF nanotubes were synthesized. These nanotubes can pass through cell membranes and thus make them useful for intracellular delivery vehicles. ${ }^{16}$ Another strategy for drug delivery application is to directly conjugate drugs to FF. ${ }^{17}$ Silva et al. ${ }^{17}$ applied this method to load rhodamine B within FF nanotubes and study its drug release kinetics and cytotoxicity. FF nanotubes also showed piezoelectric and ferroelectric properties, which makes them very interesting as functional biomaterials. ${ }^{18}$ Depending on the preparation conditions, FF can also form nanospheres rather than nanotubes. ${ }^{19}$ Gazit and co-workers ${ }^{20}$ used FF nanospheres to modify electrodes for environmental monitoring. Spoerke and coworker modified $\mathrm{FF}$ with boronic acid (BA)-functional group. ${ }^{21}$ These BA-functioned FF formed nanoribbons in response to $\mathrm{pH}$ change or adding salt.

\section{Other dipeptide nanostructures}

Dileucine (LL) can form nanotube similar to $\mathrm{FF}^{\mathbf{1 4}}$ Unlike aromatic FF peptides, aliphatic LL peptides are self-assembled into nanotubes as a result of intrinsic hydrophobic interactions. ${ }^{22}$ LL nanotubes show similar blue and green fluorescence as that of FF nanotubes. ${ }^{14}$ Kirkham et al. used fluorescein isothiocyanate (FITC)-labelled LL for bioimaging applications. ${ }^{23}$ These FITC-labelled LL showed cytocompatible and can be easily uptaken by fibroblasts. Dipeptide nanoparticles (DNPs) were exploited for bioimaging applications. Fan et al. ${ }^{11}$ reported that dipeptide WF can complex with zinc(II) ions and form DNP that can emit bright and stable fluorescence. These fluorescent nanoparticles can further be functionalized with the MUC1 aptamer (MUC is overexpressed in some cancers) and loaded with an anticancer drug doxorubicin, making them an effective 


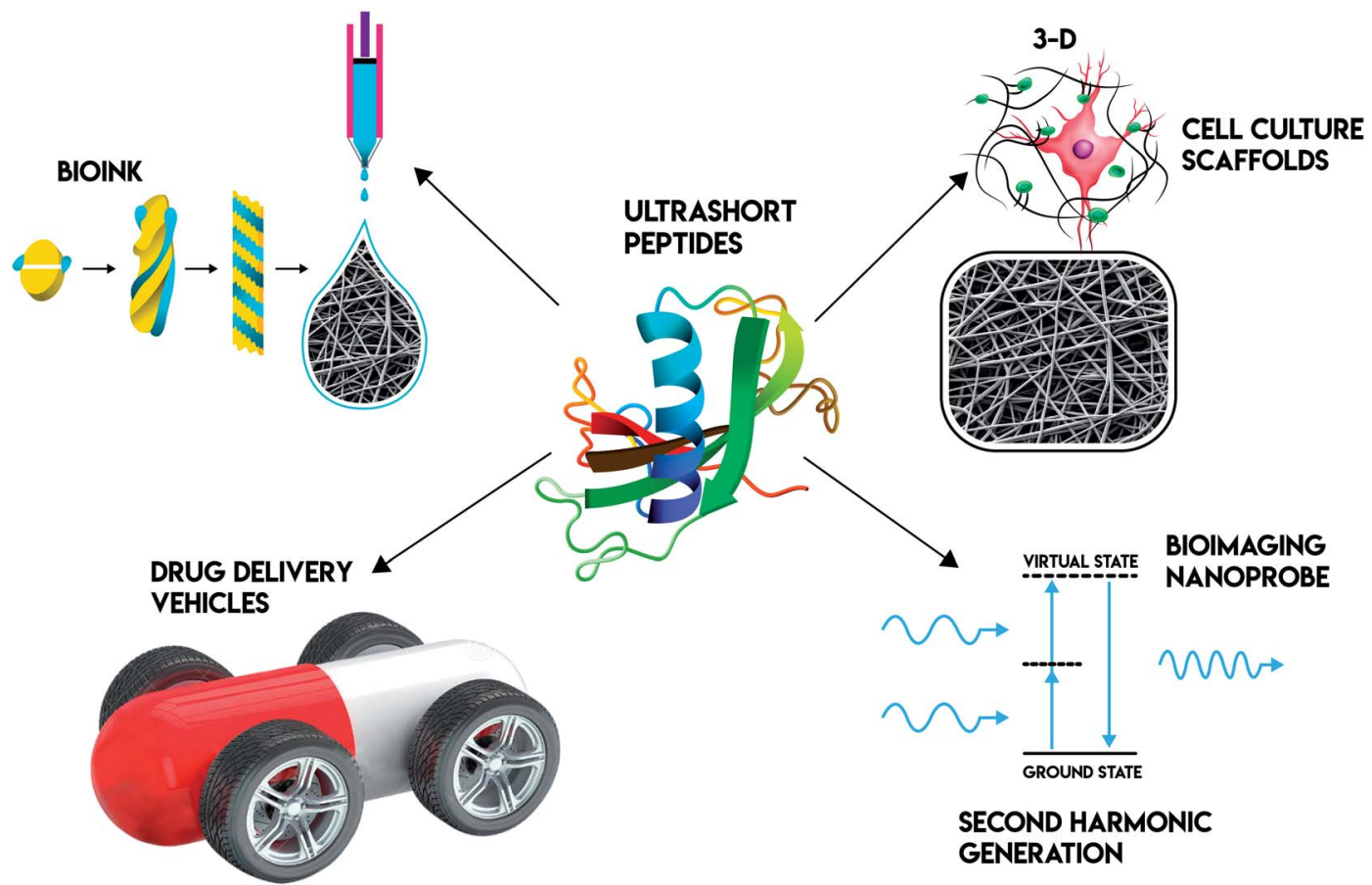

Fig. 2 Ultrashort peptide applications in bionanotechnology.

theranostic (therapeutic + diagnostic) agent. Such applications of dipeptide nanostructured materials provide us a direction for the future research and development of ultra-short peptides.

\section{FF hydrogels}

Besides nanotubes and nanospheres, fluorenylmethoxycarbonyldiphenylalanine (Fmoc-FF) can first form nanocylinders by antiparallel $\beta$-sheets stacking and then self-assemble into hydrogel..$^{24-27} \mathrm{Xu}$ and coworkers ${ }^{28}$ used enzymes to trigger and control the self assembly of small molecules (including FF) to form hydrogel. Fmoc-FF hydrogels were used as three-dimensional cell culture scaffolds. Several cell lines were used to assess the cytotoxicity of Fmoc-FF hydrogels, including Chinese hamster ovary (CHO) cell line,${ }^{26}$ bovine chondrocytes, ${ }^{27}$ human colorectal cancer (Caco) cell line, ${ }^{29}$ human cervical cancer cell line HeLa, ${ }^{29}$ and human gingival fibroblasts. ${ }^{29}$ In addition to in vitro cell assays, in vivo studies showed Fmoc $\mathrm{FF}$ hydrogel was not harmful to animals. ${ }^{29}$ Gazit and coworkers ${ }^{30}$ loaded doxorubicin and 5fluoro-uracil into Fmoc-FF hydrogel nanoparticles to study its release profile. All these results further confirmed that Fmoc-FF hydrogel can be used as biomaterials for cell culture and drug delivery applications.

\section{Other dipeptide hydrogels}

Janmey and co-workers ${ }^{31}$ reported Fmoc-LD dipeptide can form hydrogels at peptide concentrations as low as $2 \mathrm{mg} \mathrm{mL}^{-1}$ in PBS. This is the first known example of dipeptide hydrogel. It is a thermal-reversible hydrogel and was used as a carrier for the delivery of antigens. Xu and co-workers ${ }^{32}$ reported Fmoc- ${ }^{\mathrm{D}} \mathrm{A}^{\mathrm{D}} \mathrm{A}$ ( ${ }^{\mathrm{D}}$-form amino acids are unnatural) dipeptide can form hydrogels via ligand-receptor interaction. In this case, vancomycin acts as ligand, peptides serve as receptors. By varying the concentration of vancomycin, researchers can control the solgel transition process.

Ulijn and co-workers ${ }^{27}$ constructed a library of seven Fmocdipeptides (Fmoc-GG, Fmoc-AG, Fmoc-AA, Fmoc-LG, FmocFG, Fmoc-GF, Fmoc-FF) made up of the combinations of the four amino acids: G, A, L and F and examined their nanofiber dimensions, gelation behaviors and whether they can support cell culture. They found those dipeptide hydrogels were stable under cell culture conditions and can support bovine chondrocytes culture. Later on, Ulijn and co-work ${ }^{33}$ developed several types of hydrogels by mixing Fmoc-FF and Fmoc-X $(\mathrm{X}=\mathrm{K}$, or $\mathrm{S}$, or E). They found those hydrogels have different mechanical properties and different compatibility with different cell types. Fmoc-FF hydrogels showed the highest mechanical strength among those mixtures. Ulijn and co-workers ${ }^{34}$ summarized the dipeptide hydrogels in an excellent review article. Many dipeptides form hydrogels, such as Fmoc-FG, Fmoc-LG. Dipeptides often self-assemble to form $\beta$-sheets via aromatic stacking interactions and hydrogen-bonding and then aggregate into macroscopic hydrogel. Capping peptides with Fmoc functional groups plays an important role in hydrogel formation process. However, Fmoc contains aromatic ring which may lead to sideeffects in vivo. Long term biocompatibility studies of Fmoccapped peptides should be documented.

Another strategy to form dipeptide hydrogel is to use dehydro $(\Delta)$ amino acids. For example, $\Delta \mathrm{F}$ is the dehydro from of phenylalanine (F). As it can be seen in Fig. 3, its chemical structure is different from $\mathrm{F}$ by changing single bond to double bond between $\mathrm{C}_{\alpha}$ and $\mathrm{C}_{\beta}$ atoms. This change introduces conformational constraint in the peptide backbone and provides increased resistance to enzymatic degradation. 


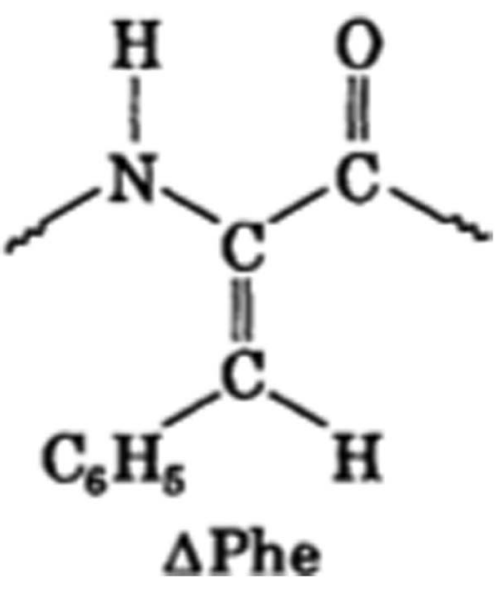

Fig. 3 The chemical structure of $\Delta \mathrm{F}$.

Dipeptides $\mathrm{F} \Delta \mathrm{F}$ forms hydrogels. ${ }^{35}$ Later on other $\Delta \mathrm{F}$ dipeptides, such as $\mathrm{L} \Delta \mathrm{F}$, also show hydrogel forming capability. ${ }^{36} \mathrm{~L} \Delta \mathrm{F}$ hydrogel is transparent, self-supportive, mechanically strong, non-toxic, injectable, proteolytically stable and responsive to external stimuli like ionic strength, $\mathrm{pH}$ and temperature. It was used to encapsulate and release various hydrophobic and hydrophilic drug molecules in a controlled manner. ${ }^{36} \Delta \mathrm{F}$ dipeptides, like $\mathrm{F} \Delta \mathrm{F}, \mathrm{I} \Delta \mathrm{F}, \mathrm{L} \Delta \mathrm{F}, \mathrm{M} \Delta \mathrm{F}$ and $\mathrm{R} \Delta \mathrm{F}$, can also form nanoparticles and be used as drug carriers loaded with various of drug-like molecules..$^{37,38}$ Another interesting strategy to use dipeptide hydrogel as drug delivery vehicles is to directly complex dipeptides with small molecular drug. For example, 5fluorouracil (5-Fu) is an antimetabolite drug and it can selfassemble with Fmoc-KK dipeptides into nanotubes or nanofibers and then form hydrogel. ${ }^{39}$ Their results showed 5-Fu was released in a sustained manner and able to kill three types of human cancer cells in vitro (human non-small cell lung cancer cell lines A549, H460, and H23).

\section{Tripeptides}

\section{Tripeptide nanostructures}

FFF tripeptide building blocks form larger sizes of $\beta$-sheet-like structures (up to 13 chains) in comparison to $\mathrm{FF}$ dipeptide assembly (up to 7 chains). It can form nanorods, nanospheres, nanotubes and nanovesicles, based on a molecular dynamic simulation study. ${ }^{40} \mathrm{FFF}$ nanobelts and nanospheres also showed second-order nonlinear optical response ${ }^{12}$ and visible (blue and green) fluoresce. ${ }^{14}$

\section{Tripeptide hydrogels}

Several tripeptides were found to be able to form hydrogels in water. Chronopoulou et al. ${ }^{41}$ used Fmoc-FFF as cell culture scaffolding materials. Marchesan et al. ${ }^{42}$ found that uncapped tripeptides ${ }^{\mathrm{D}} \mathrm{VFF}$ and ${ }^{\mathrm{D}} \mathrm{FFV}$ ( $\left({ }^{\mathrm{D}}\right.$ is un-natural form of peptide) can self assemble into $\beta$-sheet fibrils that further entangle to form stiff hydrogels. On the contrary, the corresponding natural Lform peptides VFF and FFV failed to do so. This is because DVFF contains phenylalanine zippers. The $\pi-\pi$ interactions between $\mathrm{F}$ allow for molecular stacking and lead to the $\beta$-sheet secondary structure. ${ }^{43}$ Capping with functional groups is also used to as a strategy to form tripeptide hydrogel. For example, tripeptide FFA capped with tert-butyloxycarbonyl (Boc) functional group has been found to form a translucent, selfsupporting gel in aqueous solutions. ${ }^{44}$ Hauser and coworkers ${ }^{45}$ found that tripeptide IVD and IID can form hydrogels. This class of peptides contain three to seven amino acids. They share a characteristic peptide sequence: it is amphiphilic, consisting of an aliphatic amino acid tail of decreasing hydrophobicity and a hydrophilic head. These peptides self-assemble most likely via parallel-antiparallel $\alpha$-helical pair formation and subsequent stacking into fibers that condense to $\beta$-turn fibrils. ${ }^{1,45,46}$ Ulijn and co-workers ${ }^{47}$ systematically studied 8000 tripeptides and their nanostructure formation via molecular dynamic simulation. Four full L-form tripeptides, KYF, KYY, KFF and KYW, were found to form a hydrogel in the absence of organic solvents. Similarly, Hauser and co-workers ${ }^{48}$ applied both computational and experimental methods to screen a smaller sized library of tripeptides and found MYD and VIE formed hydrogels. The twenty amino acids building blocks can provide us unlimited supply of peptide biomaterials in theory.

The tripeptide RGD is known to facilitate cell adhesion, and then spreading. Consequently, it facilitates the cytoskeleton organization of osteoblasts and many cell types. Therefore, researchers are interested to develop RGD-based hydrogel scaffolds (e.g. Fmoc-RGD) for cell culture. ${ }^{49}$ However, as mentioned previously, Fmoc groups are not normally found in the ECM. Fmoc-RGD hydrogel may not be an ideal three dimensional cell culture scaffold. ${ }^{50}$ In addition, Fmoc-RGD peptide can be used as drug delivery vehicles due to FmocRGD hydrogel forming a $\beta$-sheet fibril network. It can host hydrophilic drugs. Recent report showed it can slowly release a number of molecules. ${ }^{51}$

The ubiquitous tripeptide glutathione $(\gamma$-GSH) is present in nearly all known aerobic organisms. Its oxidized disulfide form, GSSG, is shown to produce transparent, thermo-reversible gels in aqueous solutions of dimethyl sulfoxide (DMSO), dimethylformamide (DMF), and methanol. ${ }^{52}$ GSSG contains $\gamma$-glu-cys linkage and can self-assemble into an extended network of $\beta$ sheet like structures.

\section{Tetrapeptide hydrogel}

Tripeptide RGD is found in extracellular matrix (ECM) and can promote cell adhesion via integrin binding. Therefore, it is widely employed in the development of biomaterials for applications in cell culture or tissue engineering. ${ }^{53}$ Moreover, the RGDS tetrapeptide has antithrombotic activity because it is a fibrinogen recognition sequence and can inhibit platelet aggregation..$^{54}$ Fmoc-RGDS form $s \beta$-sheets and then form hydrogels. ${ }^{55}$ The hydrogel was used to culture corneal stromal fibroblasts within collagen-based substrates. ${ }^{56}$

Chronopoulou et al. ${ }^{57}$ investigated the rheological and chemico-physical properties of Fmoc-FGFF hydrogels. They showed that this hydrogel can be used to culture mammalian fibroblasts without cytotoxic effect. Luo et al. ${ }^{58}$ used Nap-GFFY$\mathrm{NMe}$ (naphthylacetic acid modified tetrapeptide of GFFY with C 
terminal methyl amide group) as vaccine adjuvants, potentially to treat human immunodeficiency virus (HIV) patients. Naskar et $a l .{ }^{59}$ found that tetrapeptide GAIL and tetrapeptide GFIL, without having any capping groups, can form hydrogels at physiological $\mathrm{pH}$. They also investigated the potential of these hydrogels as drug delivery vehicles.

Gao et al. reported tetra-peptide, YYYY, can be used as a controlled release vehicle for anti-cancer drugs. ${ }^{60} \mathrm{Ac}^{-} \mathrm{YYY}_{p} \mathrm{Y}$ OMe $(\mathrm{Ac}=\mathrm{N}$-terminal acetylation, $\mathrm{OMe}=\mathrm{C}$-terminal methyl ester group) is a phosphatase responsive peptide and can form supramolecular hydrogel via enzyme catalyzed molecular selfassembly. The symbol $p$ denotes phosphorylated peptide. Similarly, Nap-GFF $p$-OMe (Nap = naphthyl) forms enzymatic responsive hydrogel. ${ }^{61}$ These hydrogels could be used for cell cultures, tissue engineering, and drug delivery. Recently, the same group reported Nap- $G^{D} F^{D} F^{D} Y$ hydrogel that is responsive to $\mathrm{CD}^{+} \mathrm{T}$ cells, which could lead to new vaccines against infection diseases and cancers. ${ }^{62}$

Similar to tripeptides, Hauser and coworkers ${ }^{45}$ found that uncapped IVAD, IIID and IIIK can form hydrogels. However, mainly hexapeptides were studied in their group for various applications (i.e. wound dressing and drug delivery) in bionanotechnology. Hexapeptides are beyond the scope of this review.

\section{Applications of ultrashort peptides in bionanobiotechnology}

\section{Ultrashort peptides for cell culture}

Peptide based biomaterial are intriguing because they can be $d e$ novo designed at molecular level to mimic the natural tissue. ${ }^{1}$ As it can be seen in Table 1, one of the most common applications in bionanotechnology for ultrashort peptides is for cell culture. From the very beginning when researchers started to manipulate cell in vitro, various materials have been used, such as glass, tissue culture polystyrene (TCPS) and silicon wafers. To make these cell culture substrates suitable for growing various types of cells, it is important to introduce cell adhesive cues to these substrates to guide cell attachment and maintain their functions. Typically, extracellular matrix protein, such as fibronectin and laminin, are applied as surface coatings to improve cell adhesion. Amino acid sequences of RGD and YIGSR are derived from fibronectin and laminin. ${ }^{63}$ Instead of immobilizing the whole protein, researchers prefer to immobilize short peptides on the cell culture substrates as it is easy to control these peptides' orientation on culture substrate at a lower cost. As mentioned previously, both tripeptide RGD and tetrapeptide RGDS hydrogels were used for cell culture. ${ }^{50,55}$ However, using peptide hydrogels is still expensive in comparison to immobilizing peptides on the existing cell culture substrates. Alternatively, researchers are using self-assembling peptide amphiphiles (PA) as biomaterials for cell culture. ${ }^{64} \mathrm{PA}$ is not covered in this review. Other ultrashort peptide hydrogels such as FF and FFF support many types of cell growth. ${ }^{26,27,29,41}$ One thing worth noting is that FF is a core recognition motif of the $\beta$-amyloid polypeptide but is not present in extracellular matrix. This limits their in vivo bionanotechnology applications.
Table 1 Types of ultrashort peptides and their applications

\begin{tabular}{lll}
\hline Type of peptide & Type of applications & Reference \\
\hline Dipeptide FF & Bioelectric wires & 7 \\
& Bioimaging nanoprobes & $12-14$ \\
& Cell culture scaffolds & 26,27 and 29 \\
& Drug delivery vehicles & 16,17 and 30 \\
& 2D printing inks & 69 \\
Dipeptide LD & Drug delivery vehicles & 31 \\
Dipeptide LL & Bioimaging probes & 14 \\
Dipeptide WF & Theranostic agents & 11 \\
Dipeptide F $\Delta$ F & Drug delivery vehicles & 35 \\
Dipeptide L $\Delta$ F & Drug delivery vehicles & 36 \\
Tripeptide FFF & Cell culture scaffolds & 41 \\
Tripeptide FFF & Bioimaging nanoprobes & 12 and 14 \\
Tripeptide RGD & Cell culture scaffolds & 50 \\
& Drug delivery vehicles & 51 \\
Tetrapeptide RGDS & Cell culture scaffolds & 56 \\
& (with collagen) & \\
Tetrapeptide FGFF & Cell culture scaffolds & 57 \\
Tetrapeptide GFFY & Vaccine adjuvant & 58 \\
Tetrapeptide GAIL & Drug delivery vehicles & 59 \\
Tetrapeptide GFIL & & \\
& & \\
& &
\end{tabular}

\section{Ultrashort peptides for drug delivery}

Ultrashort peptides are commonly used as vehicles for drug delivery (Table 1). Drug delivery basically faces two hurdles: ${ }^{.5}$ targeting both in vitro and in vivo. Ultrashort peptides are a perfect choice in this regard. For example, Fan et al. ${ }^{\mathbf{1 1}}$ used WF dipeptide functioned with the MUC1 aptamer and doxorubicin to target cancer cells. Sun et al. ${ }^{39}$ directly conjugated 5-Fu (an antimetabolite drug) to a ultrashort peptide and achieved sustained release over time. Peptide can form various nanomorphologies, such as nanotubes. Emtiazi et al. ${ }^{66}$ used diphenylalanine nanotubes (FNTs) to release $5-\mathrm{Fu}$ in a controlled release manner. Ultrashort peptide hydrogels can be designed to encapsulate hydrophobic drug molecules through physical or covalent bonds. ${ }^{67}$ One problem associated with peptide-based drug delivery vehicles is their susceptibility to enzymatic degradation in vivo. ${ }^{67}$ As mentioned previously, introducing the dehydro form of phenylalanine, $\Delta \mathrm{F}$ can provide resistance against enzymatic degradation. Another strategy to solve this problem is to partially substitute L-form to D-form amino acids. ${ }^{32,68}$ As shown in Table 1, several dipeptides, FF, $\mathrm{LD}, \mathrm{F} \Delta \mathrm{F}, \mathrm{L} \Delta \mathrm{F}$ and tripeptide RGD were successfully used as drug delivery vehicles.

\section{Ultrashort peptides for bioprinting}

Inkjet printing, present in households and most offices, is a common method for transferring digital data to paper or transparencies. As previously discussed, the aromatic dipeptides, FF, can form both spherical and tubular nanostructures. These structures were used as bioink to be efficiently printed on surfaces via a desktop inkjet printer (two-dimensional, 2D). ${ }^{69}$ Previously, it was shown that a variety of cells can be encapsulated into the peptide hydrogel. Therefore, in principle, cellcontaining peptide hydrogels can be printed on the surface either by soft lithography or inkjet printing (i.e. bioprinting). In 

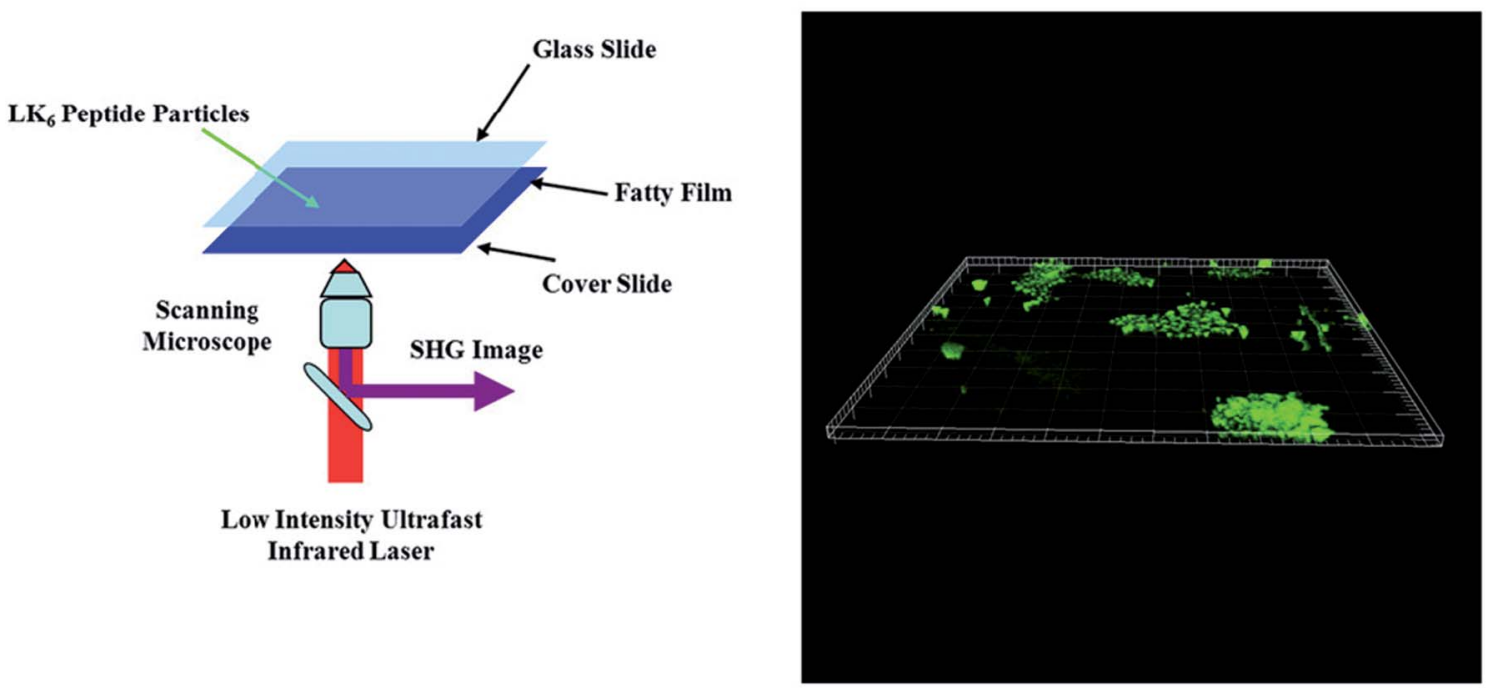

Fig. 4 Schematic illustration of an experimental set-up showing peptide nanoparticles with fat tissue from a rat, sandwiched between glass slides (left). 3D image of the peptide nanoparticles as a SHG z-stack (right). Figure reproduced from ref. 10. Copyright 2016 IOP Publishing.

2015, Loo et al. ${ }^{70}$ reported using ultrashort peptide hydrogels as bioink for three-dimensional (3D) bioprinting. These peptide hydrogels undergo instantaneous gelation under physiological conditions, resulting in peptide hydrogel scaffolds. Furthermore, the hydrogel scaffolds support long-term 3D cultures of encapsulated human stem cells, as well as primary cells. The cells were further differentiated and grew into organotypic structures (skin and small intestine), which could be a useful platform for drug screening and diagnostics. Although hexapeptides were used by Loo et al., in principle, di-tri- and tetrapeptides can be used as bioink as well. Recently, Arab et al. used two tetra-peptides, $\mathrm{CH}-01$ and $\mathrm{CH}-02$, as bioinks for 3D culture of $\mathrm{C} 2 \mathrm{C} 12$ mouse myoblasts. ${ }^{71}$

\section{Bioimaging nanoprobes}

Bioimaging refers to any imaging technique used in life sciences. It uses light, fluorescence, electrons, X-rays, ultrasound, magnetic resonance as sources for imaging. Among them, optical imaging techniques are widely used because they provide high-resolution, three dimensional images at cellular and sub-cellular level. ${ }^{72-74}$ Fluorescence microscopy is commonly used as it provides structural and dynamic information about biological systems at single cell level. However, it relies on labelling with fluorescent probes. ${ }^{72}$ Label-free optical imaging techniques are thus superior to the conventional fluorescence-based imaging technique. Second harmonic generation ( $\mathrm{SHG}$ ) is such a label-free technique. SHG was demonstrated by Franken et al. in $1961 .^{75}$ It is a nonlinear optical (NLO) process in which two incident photons $\left(\omega_{1}\right)$ interact with an NLO medium, and then are converted into one new photon. This combination generates a new photon with twice the energy, twice the frequency $\left(2 \omega_{1}\right)$, and half the wavelength $\left(\lambda_{\mathrm{SHG}}=\lambda / 2\right)$ of the original photons. Fibrillar collagen is the most abundant protein in mammals and is extremely bright under SHG microscopy. ${ }^{76}$ Previous studies have shown that SHG can be used to differentiate collagens subtypes (i.e. type I vs. type II) and discriminate myosin and collagen. ${ }^{74}$ SHG has been successfully applied in diagnosis diseases, such as liver fibrosis and epithelial cancers. ${ }^{74}$ Other than collagens peptides can be seen under an SHG microscope. Aromatic ultrashort peptides, such as FF and FFF, are SHG-active. ${ }^{\mathbf{1 2 - 1 4}}$ Besides these, aliphatic ultrashort peptides showed similar brightness as collagen type I under an SHG microscope (Fig. 4). ${ }^{10}$ In the near future, we expect more ultrashort peptides to be applied as bioimaging nanoprobes for SHG or other non-linear optical microscopy. Koizumi et al. ${ }^{77}$ showed dipeptide derivatives have strong SHG signals and these materials can be used as electro-optical switch. Besides the development of nanoprobes for non-linear optical microscopy, ultrashort peptides can be used photonic crystals. Arnon et al. reported that fluorenylmethoxycarbonyl$\beta, \beta$-diphenyl-A-OH amino acid can form colorful crystals, similar to opal. ${ }^{78}$

\section{Perspective}

In this review article, we discussed three types of ultrashort peptides (dipeptides, tripeptides and tetrapeptides, $(\leq 4$ amino acids)). They can form various types of nanostructures and possess interesting electrical, mechanical and optical properties. These ultrashort peptides can be applied as bioimaging probes, bioprinting ink, tissue culture scaffolds, and drug delivery vehicles. One of the future directions is to develop theranostic agents. These agents serve as both bioimaging probes and molecular therapeutics (i.e. DNPs). In the near future, more studies are expected to be done in this direction. Another interesting topic is to design new peptide-based biomaterials. Such work relies on computational simulation and experimental validation (e.g. works from Ulijn and coworkers, Hauser and co-workers). Besides the well-studied FF, more ultrashort peptides are emerging in the field of peptide science and biomaterials. The ultrashort peptides discovered by Hauser and co-workers are an excellent example in this regard. 
These ultrashort peptides are purely synthetic, cost-effective, and easy to make. These peptide-based biomaterials are nonhemolytic, non-immunogenic, and can support many cell types growth and differentiation. They have been successfully applied as cell culture scaffolds, 3D bio-printing ink, bioimaging probes, and vaccine adjuvants. More applications of these peptide biomaterials can be expected in the near future.

\section{Conflicts of interest}

There are no conflicts to declare.

\section{Acknowledgements}

This project was supported by the National Key Basic Research Program of China (2015CB352006), the National Natural Science Foundation of China (81771881), the Program for Changjiang Scholars and Innovative Research Team in University (IRT_15R10), the Natural Science Foundation of Fujian Province (2018J07004), the Special Funds of the Central Government Guiding Local Science and Technology Development (2017L3009), the Science and Technology Planning Project of Guangdong Province (2016A020220014), and the Fujian Provincial Youth Top-notch Talent Support Program.

\section{References}

$1 \mathrm{M} . \mathrm{Ni}$ and C. A. E. Hauser, Self-Assembled Peptide Nanostructures for Regenerative Medicine and Biology, in Micro and Nanofabrication Using SelfAssembled Biological Nanostructures, ed. J. C.-L. E. Svendsen, William Andrew Publishing, Oxford, 2015, Ch. 4, pp. 63-90.

2 H. Cui, T. Muraoka, A. G. Cheetham and S. I. Stupp, Selfassembly of giant peptide nanobelts, Nano Lett., 2009, 9, 945-951.

3 U. Khoe, Y. Yang and S. Zhang, Self-assembly of nanodonut structure from a cone-shaped designer lipid-like peptide surfactant, Langmuir, 2009, 25, 4111-4114.

4 D. E. Wagner, C. L. Phillips, W. M. Ali, G. E. Nybakken, E. D. Crawford, A. D. Schwab, et al. Toward the development of peptide nanofilaments and nanoropes as smart materials, Proc. Natl. Acad. Sci. U. S. A., 2005, 102, 12656-12661.

5 R. Orbach, L. Adler-Abramovich, S. Zigerson, I. MironiHarpaz, D. Selikta and E. Gazit, Self-Assembled FmocPeptides as a Platform for the Formation of Nanostructures and Hydrogels, Biomacromolecules, 2009, 10, 2646-2651.

$6 \mathrm{~W}$. Wang and Y. Chau, Self-assembled peptide nanorods as building blocks of fractal patterns, Soft Matter, 2009, 5, 4893-4898.

7 M. Reches and E. Gazit, Casting metal nanowires within discrete self-assembled peptide nanotubes, Science, 2003, 300, 625-627.

8 S. Vauthey, S. Santoso, H. Gong, N. Watson and S. Zhang, Molecular self-assembly of surfactantlike peptides to form nanotubes and nanovesicles, Proc. Natl. Acad. Sci. U. S. A., 2002, 99, 5355-5360.
9 M. J. Webber, E. A. Appel, E. W. Meijer and R. Langer, Supramolecular biomaterials, Nat. Mater., 2016, 15, 13-26.

10 E. D. Fabrizio, S. Schlücker, J. Wenger, R. Regmi, H. Rigneault, C. Giuseppe, et al. Roadmap on biosensing and photonics with advanced nano-optical methods, $J$. Opt., 2016, 18, 063003.

11 Z. Fan, L. Sun, Y. Huang, Y. Wang and M. Zhang, Bioinspired fluorescent dipeptide nanoparticles for targeted cancer cell imaging and real-time monitoring of drug release, Nat. Nanotechnol., 2016, 11, 388-394.

12 A. Handelman, S. Lavrov, A. Kudryavtsev, A. Khatchatouriants, Y. Rosenberg, E. Mishina, et al. Nonlinear Optical Bioinspired Peptide Nanostructures, Adv. Opt. Mater., 2013, 1, 875-884.

13 N. Kol, L. Adler-Abramovich, D. Barlam, R. Z. Shneck, E. Gazit and I. Rousso, Self-Assembled Peptide Nanotubes Are Uniquely Rigid Bioinspired Supramolecular Structures, Nano Lett., 2005, 5, 1343-1346.

14 A. Handelman, N. Kuritz, A. Natan and G. Rosenman, Reconstructive Phase Transition in Ultrashort Peptide Nanostructures and Induced Visible Photoluminescence, Langmuir, 2016, 32, 2847-2862.

15 M. Reches and E. Gazit, Controlled patterning of aligned self-assembled peptide nanotubes, Nat. Nanotechnol., 2006, 1, 195-200.

16 X. Yan, Q. He, K. Wang, L. Duan, Y. Cui and J. Li, Transition of Cationic Dipeptide Nanotubes into Vesicles and Oligonucleotide Delivery, Angew. Chem., Int. Ed., 2007, 46, 2431-2434.

17 R. F. Silva, D. R. Araújo, E. R. Silva, R. A. Ando and W. A. Alves, L-Diphenylalanine Microtubes As a Potential Drug-Delivery System: Characterization, Release Kinetics, and Cytotoxicity, Langmuir, 2013, 29, 10205-10212.

18 V. S. Bystrov, E. Paramonova, I. Bdikin, A. Heredia, R. C. Pullar, D. Elena and A. L. Kholkin, BioFerroelectricity: Diphenylalanine Peptide Nanotubes Computational Modeling and Ferroelectric Properties at the Nanoscale, Ferroelectrics, 2012, 440, 3-24.

19 E. Gazit, Self-assembled peptide nanostructures: the design of molecular building blocks and their technological utilization, Chem. Soc. Rev., 2007, 36, 1263-1269.

20 L. Adler-Abramovich, M. Badihi-Mossberg, E. Gazit and J. Rishpo, Characterization of peptide-nanostructuremodified electrodes and their applications for ultrasensitive environmental monitoring, Small, 2010, 6, 825-831.

21 B. H. Jones, A. M. Martinez, J. S. Wheeler, B. B. McKenzie, L. L. Miller, D. R. Wheelerd and E. D. Spoerke, A multistimuli responsive, self-assembling, boronic acid dipeptide, Chem. Commun., 2015, 51, 14532-14535.

22 C. H. Gorbitz, Nanotube Formation by Hydrophobic Dipeptides, Chem.-Eur. J., 2001, 7, 23-29.

23 S. Kirkham, I. W. Hamley, A. M. Smith, R. M. Gouveia, C. J. Connon, M. Reza and J. Ruokolainen, A selfassembling fluorescent dipeptide conjugate for cell labelling, Colloids Surf., B, 2016, 137, 104-108. 
24 A. M. Smith, R. J. Williams, C. Tang, P. Coppo, R. F. Collins, M. L. Turner, et al. Fmoc-Diphenylalanine Self Assembles to a Hydrogel via a Novel Architecture Based on $\pi-\pi$ Interlocked $\beta$-Sheets, Adv. Mater., 2008, 20, 37-41.

25 R. V. Ulijn and A. M. Smith, Designing peptide based nanomaterials, Chem. Soc. Rev., 2008, 37, 66475.

26 A. Mahler, M. Reches, M. Rechter, S. Cohen and E. Gazit, Rigid, Self-Assembled Hydrogel Composed of a Modified Aromatic Dipeptide, Adv. Mater., 2006, 18, 1365-1370.

27 V. Jayawarna, M. Ali, T. A. Jowitt, A. F. Miller, A. Saiani, J. E. Gough, et al. Nanostructured hydrogels for threedimensional cell culture through self-assembly of fluorenylmethoxycarbonyl-dipeptides, Adv. Mater., 2006, 18, 611-614.

28 Y. Yang, U. Khoe, X. Wang, H. Akihiro, Y. Hidenori and S. Zhang, Designer self-assembling peptide nanomaterials, Nano Today, 2009, 4, 193-210.

29 W. T. Truong, Y. Su, D. Gloria, F. Braet and P. Thordarson, Dissolution and degradation of Fmocdiphenylalanine selfassembled gels results in necrosis at high concentrations in vitro, Biomater. Sci., 2015, 3, 298-307.

30 R. Ischakov, L. Adler-Abramovich, L. Buzhansky, T. Shekhter and E. Gazit, Peptide-based hydrogel nanoparticles as effective drug delivery agents, Bioorg. Med. Chem., 2013, 21, 3517-3522.

31 R. Vegners, I. Shestakova, I. Kalvinsh, R. M. Ezzell and P. A. Janmey, Use of a gel- forming dipeptide derivative as a carrier for antigen presentation, J. Pept. Sci., 1995, 1, 371-378.

32 Y. Zhang, H. Gu, Z. Yang and B. Xu, Supramolecular Hydrogels Respond to Ligand-Receptor Interaction, J. Am. Chem. Soc., 2003, 125, 13680-13681.

33 V. Jayawarna, S. M. Richardson, A. R. Hirst, N. W. Hodson, A. Saiani, J. E. Gough, et al. Introducing chemical functionality in Fmoc-peptide gels for cell culture, Acta Biomater., 2009, 5, 934-943.

34 S. Fleming and R. V. Ulijn, Design of nanostructures based on aromatic peptide amphiphiles, Chem. Soc. Rev., 2014, 43, 8150-8177.

35 M. Gupta, A. Bagaria, A. Mishra, P. Mathur, A. Basu, S. Ramakumar, et al. Self-Assembly of a DipeptideContaining Conformationally Restricted Dehydrophenylalanine Residue to Form Ordered Nanotubes, Adv. Mater., 2007, 19, 858-861.

36 C. K. Thota, N. Yadav and V. S. Chauhan, A novel highly stable and injectable hydrogel based on a conformationally restricted ultrashort peptide, Sci. Rep., 2016, 6, 31167.

37 S. Alam, J. J. Panda and V. S. Chauhan, Novel dipeptide nanoparticles for effective curcumin delivery, Int. J. Nanomed., 2012, 7, 4207-4222.

38 J. J. Panda, A. Kaul, S. Alam, A. K. Babbar, A. K. Mishra and V. S. Chauhan, Designed peptides as model self-assembling nanosystems: characterization and potential biomedical applications, Ther. Delivery, 2011, 2, 193-204.

39 Y. Sun, J. A. Kaplan, A. Shieh, H. L. Sun, C. M. Croce, M. W. Grinstaff, et al. Self-assembly of a 5fluorouracildipeptide hydrogel, Chem. Commun., 2016, 52, 5254-5257.
40 C. Guo, Y. Luo, R. Zhou and G. Wei, Triphenylalanine peptides self-assemble into nanospheres and nanorods that are different from the nanovesicles and nanotubes formed by diphenylalanine peptides, Nanoscale, 2014, 6, 2800-2811.

41 L. Chronopoulou, S. Lorenzoni, G. Masci, M. Dentini, A. R. Togna, G. Togna, et al. Lipasesupported synthesis of peptidic hydrogels, Soft Matter, 2010, 6, 2525.

42 S. Marchesan, C. D. Easton, F. Kushkaki, L. Waddington and P. G. Hartley, Tripeptide selfassembled hydrogels: unexpected twists of chirality, Chem. Commun., 2012, 48, 2195-2197.

43 S. Marchesan, L. Waddington, C. D. Easton, D. A. Winkler, L. Goodall, J. Forsythe, et al. Unzipping the role of chirality in nanoscale self-assembly of tripeptide hydrogels, Nanoscale, 2012, 4, 6752-6760.

44 J. Nanda, B. Adhikari, S. Basak and A. Banerjee, Formation of hybrid hydrogels consisting of tripeptide and different silver nanoparticle-capped ligands: modulation of the mechanical strength of gel phase materials, J. Phys. Chem. B, 2012, 116, 12235-12244.

45 A. Mishra, Y. H. Loo, R. H. Deng, Y. J. Chuah, H. T. Hee, J. Y. Ying, et al. Ultrasmall natural peptides self-assemble to strong temperature-resistant helical fibers in scaffolds suitable for tissue engineering, Nano Today, 2011, 6, 232239.

46 C. A. E. Hauser, R. Deng, A. Mishra, Y. Loo, U. Khoe, F. Zhuang, et al. Natural tri- to hexapeptides self-assemble in water to amyloid beta-type fiber aggregates by unexpected alphahelical intermediate structures, Proc. Natl. Acad. Sci. U. S. A., 2011, 108, 1361-1366.

47 P. W. J. M. Frederix, G. G. Scott, Y. M. Abul-Haija, D. Kalafatovic, C. G. Pappas, N. Javid, et al. Exploring the sequence space for (tri-)peptide self-assembly to design and discover new hydrogels, Nat. Chem., 2015, 7, 30-37.

48 J. Smadbeck, K. H. Chan, G. A. Khoury, B. Xue, R. C. Robinson, C. A. E. Hauser, et al. De novo design and experimental characterization of ultrashort self-associating peptides, PLoS Comput. Biol., 2014, 10, e1003718.

49 K. Chan, S. Zhuo and M. Ni, Natural and synthetic peptidebased biomaterials for bone tissue engineering, OA Tissue Engineering, 2013, 1, 6.

50 P. Worthington, D. J. Pochan and S. A. Langhans, Peptide Hydrogels - Versatile Matrices for 3D Cell Culture in Cancer Medicine, Front. Oncol., 2015, 5, 92.

51 V. Castelletto, I. W. Hamley, C. Stain and C. Connon, Slowrelease RGD-peptide hydrogel monoliths, Langmuir, 2012, 28, 12575-12580.

52 R. P. Lyon and W. M. Atkins, Self-Assembly and Gelation of Oxidized Glutathione in Organic Solvents, J. Am. Chem. Soc., 2001, 123, 4408-4413.

53 M. D. Mager, V. LaPointe and M. M. Stevens, Exploring and exploiting chemistry at the cell surface, Nat. Chem., 2011, 3, 582-589.

54 T. K. Gartner and J. S. Bennett, The tetrapeptide analogue of the cell attachment site of fibronectin inhibits platelet 
aggregation and fibrinogen binding to activated platelets, $J$. Biol. Chem., 1985, 260, 11891-11894.

55 V. Castelletto, C. M. Moulton, G. Cheng, I. W. Hamley, M. R. Hicks, A. Rodger, et al. Self-Assembly of FmocTetrapeptides Based on the RGDS Cell Adhesion Motif, Soft Matter, 2011, 7, 11405-11415.

56 R. M. Gouveia, R. R. Jones, I. W. Hamley and C. J. Connon, The bioactivity of composite Fmoc-RGDS-collagen gels, Biomater. Sci., 2014, 2, 1222-1229.

57 L. Chronopoulou, Y. Toumia, B. Cerroni, A. Gentili, G. Paradossi and C. Palocci, Biosynthesis and characterization of a novel Fmoc-tetrapeptide-based hydrogel for biotechnological applications, Colloids Surf., A, 2017, 532, 535-540.

58 Z. Luo, Q. Wu, C. Yang, H. Wang, T. He, Y. Wang, et al. A Powerful CD8 $(+)$ T-Cell Stimulating D-Tetra-Peptide Hydrogel as a Very Promising Vaccine Adjuvant, $A d v$. Mater., 2017, 29, DOI: 10.1002/adma.201601776.

59 J. Naskar, G. Palui and A. Banerjee, Tetrapeptide-Based Hydrogels: for Encapsulation and Slow Release of an Anticancer Drug at Physiological pH, J. Phys. Chem. B, 2009, 113, 11787-11792.

60 J. Gao, W. Zheng, D. Kong and Z. Yang, Dual enzymes regulate the molecular self-assembly of tetra-peptide derivatives, Soft Matter, 2011, 7, 10443-10448.

61 H. Wang, C. Ren, Z. Song, L. Wang, X. Chen and Z. Yang, Enzyme-triggered self-assembly of a small molecule: a supramolecular hydrogel with leaf-like structures and an ultra-low minimum gelation concentration, Nanotechnology, 2010, 21, 225606.

62 Z. Luo, Q. Wu, C. Yang, H. Wang, T. He, Y. Wang, Z. Wang, H. Chen, X. Li, C. Gong and Z. Yang, A Powerful CD8 ${ }^{+}$T-Cell Stimulating D-Tetra-Peptide Hydrogel as a Very Promising Vaccine Adjuvant, Adv. Mater., 2017, 29, 1601776.

63 B. D. Ratner and S. J. Bryant, Biomaterials: where we have been and where we are going, Annu. Rev. Biomed. Eng., 2004, 6, 41-75.

64 H. Cui, M. J. Webber and S. I. Stupp, Self-assembly of peptide amphiphiles: from molecules to nanostructures to biomaterials, Biopolymers, 2010, 94, 1-18.

65 A. Kakkar, G. Traverso, O. C. Farokhzad, R. Weissleder and R. Langer, Evolution of macromolecular complexity in drug delivery systems, Nat. Rev. Chem., 2017, 1, 0063.

66 G. Emtiazi, T. Zohrabi, L. Y. Lee, N. Habibi and A. Zarrabi, Covalent diphenylalanine peptide nanotube conjugated to folic acid/magnetic nanoparticles for anti-cancer drug delivery, J. Drug Delivery Sci. Technol., 2017, 41, 90-98.
67 S. Eskandari, T. Guerin, I. Toth and R. J. Stephenson, Recent advances in self-assembled peptides: Implications for targeted drug delivery and vaccine engineering, Adv. Drug Delivery Rev., 2017, 110111, 169-187.

68 R. Tugyi, K. Uray, D. Ivan, E. Fellinger, A. Perkins and F. Hudecz, Partial D-amino acid substitution: Improved enzymatic stability and preserved $\mathrm{Ab}$ recognition of a MUC2 epitope peptide, Proc. Natl. Acad. Sci. U. S. A., 2005, 102, 413-418.

69 L. Adler-Abramovich and E. Gazit, Controlled patterning of peptide nanotubes and nanospheres using inkjet printing technology, J. Pept. Sci., 2008, 14, 217-223.

70 Y. Loo, A. Lakshmanan, M. Ni, L. L. Toh, S. Wang and C. A. E. Hauser, Peptide Bioink: Self-Assembling Nanofibrous Scaffolds for 3D Organotypic Cultures, Nano Lett., 2015, 15, 6919-6925.

71 W. Arab, S. Rauf, O. Al-Harbi and C. A. E. Hauser, Novel ultrashort self-assembling peptide bioinks for 3D culture of muscle myoblast cells, Int. J. Bioprint., 2018, 4, 129.

72 A. M. Streets, A. Li, T. Chen and Y. Huang, Imaging without fluorescence: nonlinear optical microscopy for quantitative cellular imaging, Anal. Chem., 2014, 86, 8506-8513.

73 M. Ni, S. Zhuo, P. T. C. So and H. Yu, Fluorescent probes for nanoscopy: four categories and multiple possibilities, $J$. Biophotonics, 2017, 10, 11-23.

$74 \mathrm{M}$. Ni and S. Zhuo, Nonlinear optical microscopy: Endogenous signals and exogenous probes, Ann. Phys., 2015, 527, 471-489.

75 P. A. Franken, A. E. Hill, C. W. Peters and G. Weinreich, Generation of Optical Harmonics, Phys. Rev. Lett., 1961, 7, 118-119.

76 P. J. Campagnola and L. M. Loew, Second-harmonic imaging microscopy for visualizing biomolecular arrays in cells, tissues and organisms, Nat. Biotechnol., 2003, 21, 1356-1360.

77 N. Koizumi, G. Shanker, F. Araoka, K. Ishikawa, C. V. Yelamaggad and H. Takezoe, Interplay between polarity and chirality in the electric-field-responsive columnar phase of a dipeptide derivative, NPG Asia Mater., 2012, 4, e11.

78 Z. A. Arnon, D. Pinotsi, M. Schmidt, S. Gilead, T. Guterman, A. Sadhanala, S. Ahmad, A. Levin, P. Walther, C. F. Kaminski, M. Fändrich, G. S. K. Schierle, L. LadlerAbramovich, L. J. W. Shimon and E. Gazit, Opal-like Multicolor Appearance of Self-Assembled Photonic Array, ACS Appl. Mater. Interfaces, 2018, 10, 20783-20789. 